\title{
ELECTROSTATICALLY TUNABLE NANOMECHANICAL SHALLOW ARCHES
}

\author{
Syed N. R. Kazmi \\ Physical Science and \\ Engineering, King Abdullah \\ University of Science and \\ Technology, \\ Thuwal, Saudi Arabia \\ syed.kazmi@kaust.edu.sa
}

\author{
Amal Z. Hajjaj \\ Physical Science and \\ Engineering, King Abdullah \\ University of Science and \\ Technology, \\ Thuwal, Saudi Arabia \\ amal.hajjaj@kaust.edu.sa \\ Mohammad I. Younis \\ Physical Science and \\ Engineering, King Abdullah \\ University of Science and \\ Technology, \\ Thuwal, Saudi Arabia \\ mohammad.younis@kaust.edu.sa
}

\author{
Pedro M. F. J. Costa \\ Physical Science and \\ Engineering, King Abdullah \\ University of Science and \\ Technology, \\ Thuwal, Saudi Arabia \\ pedro.dacosta@kaust.edu.sa
}

\begin{abstract}
We report an analytical and experimental study on the tunability of in-plane doubly-clamped nanomechanical arches under varied DC bias conditions at room temperature. For this purpose, silicon based shallow arches are fabricated using standard e-beam lithography and surface nanomachining of a highly conductive device layer on a silicon-on-insulator (SOI) wafer. The experimental results show good agreement with the analytical results with a maximum tunability of $108.14 \%$ for $180 \mathrm{~nm}$ thick arch with a transduction gap of $1 \mu \mathrm{m}$ between the beam and the driving/sensing electrodes. The high tunability of shallow arches paves the ways for highly tunable band pass filtering applications in high frequency range.
\end{abstract}

\section{INTRODUCTION}

Micro/Nanoelectromechanical systems (MEMS/NEMS) have received considerable attention in the recent decade for their interesting advantages for several applications, such as mass/gas sensing [1,2], memory [3], logic [4], filtering [5], communications [6], gyroscopes [7], energy harvesting [8], and signal processing [9]. Tunability of MEMS/NEMS structures is highly desirable since it gives the flexibility of on-demand frequency selection for communication systems.

Many approaches have been presented to tune the resonance frequency of MEMS resonators by controlling the stiffness of the MEMS resonators with applied axial loads. Several approaches have been investigated to apply axial loads and tune the resonance frequency of a resonator, such as electrostatic [10], electromagnetic [11], and electrothermal [12]. A recent study has demonstrated large tunability of MEMS clamped-clamped straight micro beam resonators by controlling its axial load electrostatically [10] and electrothermally [12].

Since the early work of Nathanson [13], many researches have focused on the tunability of MEMS resonators by electrostatic means with applied DC bias voltage. One major drawback of the electrostatic tuning lies in its limitation of pullin instability that leads to the collapse of the resonating structure with the stationary electrode especially when a relatively higher DC voltage is applied [14]. Typically, the electrostatic force is used to induce the softening effect in straight clamped-clamped beam that results a decrease in the resonance frequency of the MEMS structure [15], except few studies where they show that the resonance frequency could be

${ }^{*}$ Corresponding Author 
tuned for higher values while increasing the DC voltage [16]. In a recent work, we have shown that a careful choice of geometric parameters, mainly the ratio between the transduction gap and the thickness, can results in considerable frequency tuning of an electrostatically driven clamped-clamped straight micro beam. Sazonova et al. [17] demonstrated experimentally the tunability (increase) of the resonance frequencies at nano scale for Carbon Nano Tube (CNT) resonators by increasing the DC bias voltage, which is considered a distinct feature of CNTs due to their high stiffness.

In this current work, we present an investigation into the tunability of NEMS shallow arches and study the effect of geometric parameters, mainly thickness of the arch and the transduction gap between the beam and the stationary electrodes under DC bias voltages. Further, the application of these arches, based on our analytical results, can be extended to tunable band pass filters for high frequency applications.

\section{PROBLEM FORMULATION}

The device under consideration, Fig. 1, consists of an initially curved clamped-clamped nano arch, which is curved due to the residual stresses from the fabrication process, actuated by a DC polarization voltage $V_{\mathrm{DC}}$ and an $\mathrm{AC}$ harmonic voltage of amplitude $V_{\mathrm{AC}}$ and frequency $\hat{\Omega}$, and is subjected to a viscous damping of coefficient $\hat{c}$. The initial shape $\hat{w}_{0}(x)$ is governed by (1) [18].

$$
\hat{w}_{0}(\hat{x})=-\frac{1}{2} \hat{b}_{0}\left(1-\cos \left(2 \pi \frac{\hat{x}}{l}\right)\right.
$$

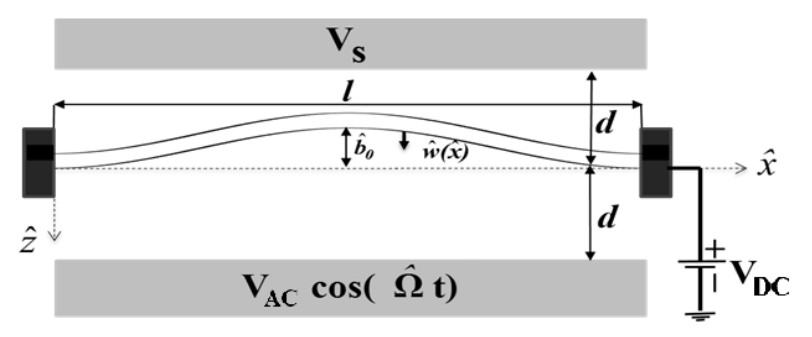

Fig. 1: Schematic of an electrostatically actuated clampedclamped shallow arch.

The nonlinear Euler-Bernoulli equation of motion governing the transverse deflection of the $\operatorname{arch} \hat{w}(\hat{x}, \hat{t})$ is written as follows [18]:

$$
\begin{gathered}
\rho b h \frac{\partial^{2} \hat{w}}{\partial \hat{t}^{2}}+\hat{c} \frac{\partial \hat{w}}{\partial \hat{t}}+E I \frac{\partial^{4} \hat{w}}{\partial \hat{x}^{4}}=\left(\frac{\partial^{2} \hat{w}}{\partial \hat{x}^{2}}+\frac{d^{2} \hat{w}_{0}}{d \hat{x}^{2}}\right) \\
\cdot\left[\hat{N}+\frac{E A}{2 l} \int_{0}^{l}\left(\left(\frac{\partial \hat{w}}{\partial \hat{x}}\right)^{2}+2 \frac{\partial \hat{w}}{\partial \hat{x}} \frac{d \hat{w}_{0}}{d \hat{x}}\right) d \hat{x}\right] \\
+\frac{1}{2} \varepsilon b \frac{\left(V_{D C}+V_{A C} \cos (\hat{\Omega} t)\right)^{2}}{\left(d-\hat{w}-\hat{w}_{0}\right)^{2}}+\frac{1}{2} \varepsilon b \frac{\left(V_{D C}-V_{S}\right)^{2}}{\left(d+\hat{w}+\hat{w}_{0}\right)^{2}}
\end{gathered}
$$

The nano arch is subjected to the following boundary conditions:

$$
\hat{w}(0, \hat{t})=\hat{w}(l, \hat{t})=0 \text { and }\left.\frac{\partial \hat{w}}{\partial \hat{x}}\right|_{(0, \hat{t})}=\left.\frac{\partial \hat{w}}{\partial \hat{x}}\right|_{(l, \hat{t})}=0
$$

where $\hat{x}$ is the position along the nano arch, $\hat{t}$ is time, $l, b$ and $h$ are, respectively, the length, depth, and thickness of the nano arch, $\rho$ is the material density, $I=b h^{3} / 12$ is the moment of inertia of the rectangular cross-section of area $A=b h, E$ is Young's modulus, $N$ is the residual axial load, $d$ is the gap between the nano arch and the stationary electrode, and $\varepsilon$ is the dielectric constant of the medium. The last term represents the electrostatic force from the sensing electrode. $V_{\mathrm{S}}$ is the DC voltage applied to the sensing electrode, which is taken in this work equal to zero.

For convenience, we introduce the nondimensional variables as below

$$
\begin{aligned}
& \quad w=\frac{\hat{w}}{d} ; x=\frac{\hat{x}}{l} ; t=\frac{\hat{t}}{T} ; b_{0}=\frac{\hat{b}_{0}}{d} \\
& \text { and } w_{0}=\frac{\hat{w}_{0}}{d}=\frac{1}{2} b_{0}(1-\cos (2 \pi x))
\end{aligned}
$$

where $T=\sqrt{\rho b h l^{4} / E I}$ is a time scale. Substituting (4) into (2) and (3), we obtain the nondimensional equation of motion of the beam

$$
\begin{gathered}
\frac{\partial^{2} w}{\partial t^{2}}+c \frac{\partial w}{\partial t}+\frac{\partial^{4} w}{\partial x^{4}}=\left(\frac{\partial^{2} w}{\partial x^{2}}+\frac{d^{2} w_{0}}{d x^{2}}\right) \\
\cdot\left[N+\alpha_{1} \int_{0}^{1}\left[\left(\frac{\partial w}{\partial x}\right)^{2}+2 \frac{\partial w}{\partial x} \frac{d w_{0}}{d x}\right] d x\right] \\
+\alpha_{2} \frac{\left(V_{D C}+V_{A C} \cos (\Omega t)\right)^{2}}{\left(1-w-w_{0}\right)^{2}}-\alpha_{2} \frac{V_{D C}{ }^{2}}{\left(1+w+w_{0}\right)^{2}}
\end{gathered}
$$

subjected to the nondimensional boundary conditions

$$
w(0, t)=w(1, t)=0 \text { and }\left.\frac{\partial w}{\partial x}\right|_{(0, t)}=\left.\frac{\partial w}{\partial x}\right|_{(1, t)}=0
$$

The nondimensional (except for $\alpha_{2}$ ) parameters appearing in (5) are defined as below

$$
\alpha_{1}=6\left(\frac{d}{h}\right)^{2} ; \alpha_{2}=\frac{6 \varepsilon l^{4}}{E h^{3} d^{3}} ; N=\frac{l^{2}}{E I} \hat{N} ; c=\frac{l^{4}}{E I T} \hat{c} \text { and } \Omega=T \hat{\Omega}
$$

The deflection of the nano arch under the electrostatic force is split into a static deflection $w_{s}(x)$ due to $V_{\mathrm{DC}}$ and a small dynamic deflection as below

$$
w(x, t)=w_{s}(x)+w_{d}(x, t)
$$


To determine the static deflection $w_{s}(x)$ we set the time derivatives as well as the $\mathrm{AC}$ force equal to zero, which yields

$$
\begin{gathered}
\frac{d^{4} w_{s}}{d x^{4}}=\left(\frac{d^{2} w_{s}}{d x^{2}}+\frac{d^{2} w_{0}}{d x^{2}}\right)\left[N+\alpha_{1} \int_{0}^{1}\left(\left(\frac{d w_{s}}{d x}\right)^{2}\right.\right. \\
\left.\left.+2 \frac{d w_{s}}{d x} \frac{d w_{0}}{d x}\right) d x\right]+\alpha_{2} \frac{V_{D C}{ }^{2}}{\left(1-w_{s}-w_{0}\right)^{2}} \\
-\alpha_{2} \frac{V_{D C}{ }^{2}}{\left(1+w_{s}+w_{0}\right)^{2}}
\end{gathered}
$$

with the associated boundary conditions

$$
w_{S}(0)=w_{s}(1)=0 \text { and }\left.\frac{d w_{s}}{d x}\right|_{x=0}=\left.\frac{d w_{S}}{d x}\right|_{x=1}=0
$$

The linearized equation of motion describing the small dynamic behavior of the shallow arch around the deflected shape is derived by substituting equation (8) into equation (6) and dropping the terms representing the equilibrium position, equations (9), and the nonlinear terms. These yield

$$
\begin{aligned}
& \frac{\partial^{2} w_{d}}{\partial t^{2}}+\frac{\partial^{4} w_{d}}{\partial x^{4}}=\left[N+\alpha_{1} \int_{0}^{1}\left[\left(\frac{d w_{s}}{d x}\right)^{2}+2 \frac{d w_{s}}{d x} \frac{d w_{0}}{d x}\right] d x\right] \frac{\partial^{2} w_{d}}{\partial x^{2}} \\
& +2 \alpha_{1} \int_{0}^{1}\left[\left(\frac{d w_{s}}{d x}+\frac{d w_{0}}{d x}\right) \frac{\partial w_{d}}{\partial x}\right] d x\left(\frac{d^{2} w_{s}}{d x^{2}}+\frac{d^{2} w_{0}}{d x^{2}}\right) \\
& +2 \alpha_{2} \frac{\left(V_{D C}+V_{A C} \cos (\Omega t)\right)^{2}}{\left(1-w_{s}-w_{0}\right)^{3}} w_{d}+2 \alpha_{2} \frac{V_{D C}{ }^{2}}{\left(1+w_{s}+w_{0}\right)^{3}} w_{d}
\end{aligned}
$$

with the associated boundary conditions

$$
w_{d}(0, t)=w_{d}(1, t)=0 \text { and }\left.\frac{\partial w_{d}}{\partial x}\right|_{x=0, t}=\left.\frac{\partial w_{d}}{\partial x}\right|_{x=1, t}=0
$$

To solve the static problem and equation (9), we refer to the Galerkin procedure in which we use the undamped linear mode shapes of a straight micro beam as basis functions. Therefore the deflection is expressed as

$$
w_{s}(x)=\sum_{i=0}^{n} \mathrm{k}_{i} \kappa_{i}(x)
$$

where $k_{i}(i=0,1,2 \ldots n)$ denotes the nondimensional modal coordinates and $\kappa_{\mathrm{i}}(\mathrm{x})(i=0,1,2 \ldots n)$ denotes the undamped mode shapes of the unactuated shallow arch governed by

$$
\frac{d^{4} \kappa_{i}}{d x^{4}}-\omega_{i}^{2} \kappa_{i}=2 \alpha_{1} \frac{d^{2} w_{0}}{d x^{2}} \int_{0} \frac{d \kappa_{i}}{d x} \frac{d w_{0}}{d x} d x
$$

with the associated boundary conditions:

$$
\kappa_{i}(0)=\kappa_{i}(1)=0 \text { and }\left.\frac{d \kappa_{i}}{d x}\right|_{x=0}=\left.\frac{d \kappa_{i}}{d x}\right|_{x=1}=0
$$

where $\omega_{\mathrm{i}}$ represents the $i^{\text {th }}$ natural frequency of the nano arch.

To determine the variation of the natural frequency of the nano arch under the DC polarization voltage, we resort also to the Galerkin discretization to solve the eigenvalue problem [18]. Toward this, we let:

$$
w_{d}(x, t)=\sum_{i=0}^{n} q_{i}(t) \kappa_{i}(x)
$$

where $\mathrm{q}_{\mathrm{i}}(\mathrm{t})(i=0,1,2 . . n)$ denotes the nondimensional modal coordinates and $\kappa_{\mathrm{i}}(\mathrm{x}) \quad(i=0,1,2 . . n)$ denotes the unactuated shallow arch governed by equation (14).

Then, we substitute equation (16) into equation (11), multiplying the outcome by the mode shape $\kappa_{\mathrm{j}}$ and integrating over the arch domain (from 0 to 1 ), which yields the below equation [18]

$$
\begin{aligned}
\ddot{q}_{j} & =\left[2 \alpha_{1} \int_{0}^{1} \kappa_{j} \frac{\partial^{2} w_{s}}{\partial x^{2}} d x \int_{0}^{1}\left(\sum_{i=0}^{n} q_{i} \kappa_{i} \frac{\partial w_{s}}{\partial x}\right) d x\right]+\left[\alpha_{1} \int_{0}^{1}\left(\frac{\partial w_{s}}{\partial x}\right)^{2} d x\right] \\
& \cdot \int_{0}^{1} \kappa_{j}\left(\sum_{i=0}^{n} q_{i} \kappa_{i}^{\prime \prime}\right) d x+\int_{0}^{1} \kappa_{j} \frac{2 \alpha_{2} V_{D C}{ }^{2}}{\left(1-w_{s}-w_{0}\right)^{3}}\left(\sum_{i=0}^{n} q_{i} \kappa_{i}\right) d x \\
& +\int_{0}^{1} \kappa_{j} \frac{2 \alpha_{2} V_{D C}{ }^{2}}{\left(1+w_{s}+w_{0}\right)^{3}}\left(\sum_{i=0}^{n} q_{i} \kappa_{i}\right) d x
\end{aligned}
$$

Then, we compute the Jacobin of the system for each value of $V_{\mathrm{DC}}$ and find the corresponding resonance frequency by taking the square root of its eigenvalues.

\section{FABRICATION AND MEASUREMENTS}

The shallow arches with gap to thickness ratio of more than 4 are fabricated from a $<100>$ highly- conductive boron doped Si device layer of silicon-on-insulator (SOI) wafer. The fabrication process employ standard e-beam lithography and surface nanomachining techniques. The main fabrication steps are illustrated in Fig. 2. The fabrication starts with cleaning of SOI wafer by dipping it in a piranha solution, heated at $120{ }^{\circ} \mathrm{C}$, followed by a deionized (DI) water rinse to remove the organic residues. The native oxide is removed by using $1 \% \mathrm{HF}$ solution until the surface turns to hydrophobic followed by a deionized (DI) water rinse and spin drying, Fig. 2a. The thickness of the device layer is reduced from $2 \mu \mathrm{m}$ to $850 \mathrm{~nm}$ by thermal oxidation of the SOI wafer, Fig $2 \mathrm{~b}$, and then removing the thermally grown oxide using buffered oxide etch (BOE), Fig 2c. In order to achieve dimensions in nanometers range the e-beam lithography is employed to pattern 950 PMMA A4 positive tone e-beam resist, Fig. 2d. Reactive ion etching (RIE) is performed at $-10{ }^{\circ} \mathrm{C}$ to separate the nano beam, anchoring pads, and the $\mathrm{RF}$ electrodes from the rest of wafer surface. This entire wafer surface will serve as ground during the characterization of fabricated devices, Fig. 2e. Finally, the HF vapor etch is done to have free standing nano beams by removing the underneath $\sim 1 \mu \mathrm{m}$ box layer, Fig. 2f. The beams are approximately $15 \mu \mathrm{m}$ long $(l), 850 \mathrm{~nm}$ deep $(b), 130 \mathrm{~nm}$ and $180 \mathrm{~nm}$ thick $(h)$ with $850 \mathrm{~nm}$ and $1 \mu \mathrm{m}$ transduction gaps $(d)$ between the beam and the driving/sensing electrodes.

Fig. 3 shows an SEM image of a shallow arch fabricated using the fabrication process flow of Fig. 2. The beam is approximately $15 \mu \mathrm{m}$ long, $130 \mathrm{~nm}$ thick, and $850 \mathrm{~nm}$ deep with a transduction gap of $1 \mu \mathrm{m}$ between the arch and the 
driving/sensing electrodes. Note that the beam is slightly curved due to the residual stress in the device layer. Therefore, thinner beams are prone to relatively higher initial curvature than the thicker beams after HF vapor release. The observed initial rise ranges from $\sim 100 \mathrm{~nm}$ to $\sim 50 \mathrm{~nm}$ for beams of thickness $130 \mathrm{~nm}$ and $180 \mathrm{~nm}$, respectively.

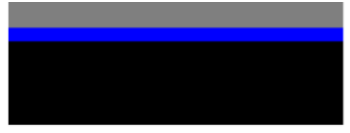

Substrate preparation

(a)

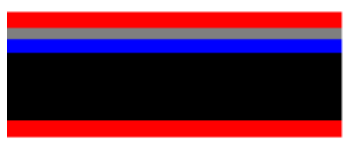

Thermal oxidation

(b)

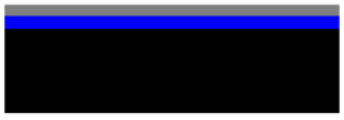

Buffered oxide etching

(c)

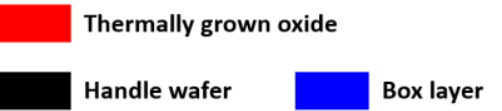

Fig. 2: Process flow for nano arches (a) Substrate cleaning and native oxide removal. (b) Thermal oxidation to thin down the device layer. (c) BOE treatment to remove the thermally grown oxide. (d) Patterning of e-beam resist (950 PMMA A4). (e) RIE to fabricate the nano arches and to separate the entire device structure from the ground plane. (f) HF vapor etching for free standing nano arches.

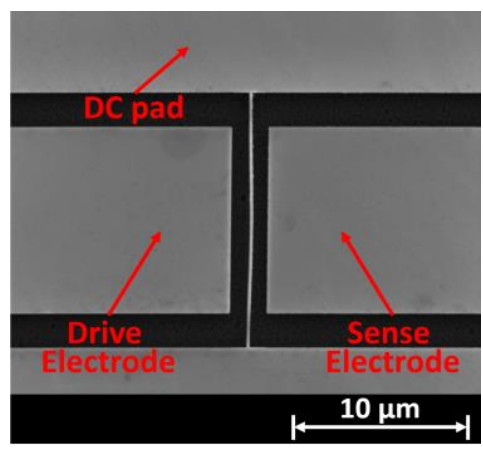

Fig. 3: An SEM image of an arch having a length $(l)$ of $15 \mu \mathrm{m}$, depth $(b)$ of $850 \mathrm{~nm}$, and thickness $(h)$ of $130 \mathrm{~nm}$ with a transduction gaps (d) of $1 \mu \mathrm{m}$ between the beam and the driving/sensing electrodes.

The electrical characterization of the fabricated devices is performed in an ST-500 JANIS probe station, Fig. 4a, capable of measuring the arch response under controlled vacuum and varied chuck temperature conditions. The $\mathrm{S}_{21}$ transmission parameter, frequency response, is measured through Agilent E5071C network analyzer. Fig. 4b shows the photograph of the nano arch under test inside the probe station at room temperature.

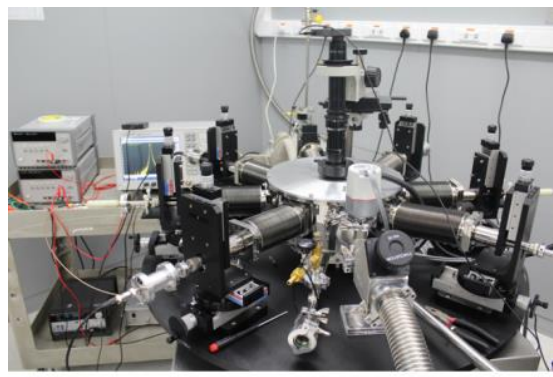

(a)

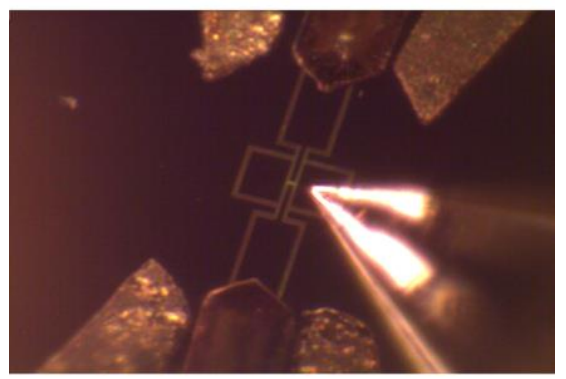

(b)

Fig. 4: (a) Photograph of ST-500 JANIS probe station having 2 RF and 4 DC probe arms. (b) Snapshot of a nano arch under test.

Fig. 5 shows schematic of the driving and sensing configuration of the resonator in its two-port. The arch is excited by applying a DC bias voltage on it and an ac signal from one of the port of the network analyzer (Agilent E5071C) to one of its electrodes, named as driving electrode. The motional current is sensed through the other electrode, named as sensing electrode, and is fed into a low-noise amplifier (LNA) with its output coupled to the other port of the network analyzer.

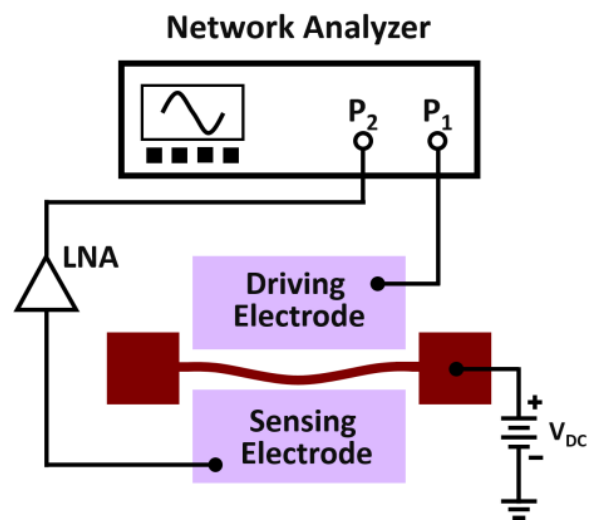

Fig. 5: A schematic of the measurement setup in its two-port driving and sensing configuration with DC polarization voltage applied to the nano arch. 


\section{RESULTS AND DISCUSSION}

Fig. $6 \mathrm{a}$ and Fig. $6 \mathrm{~b}$ show the variation of the resonance frequency of two nano arches of $15 \mu \mathrm{m}$ length, $850 \mathrm{~nm}$ depth, and $130 \mathrm{~nm}$ thickness with the transduction gaps of $850 \mathrm{~nm}$ and $1 \mu \mathrm{m}$ between the beam and the driving/sensing electrodes for varied DC polarization voltage. The figures compare the analytical and experimental data. The analytical results are obtained by considering the effective values as in Table I, which are slightly different from the designed nominal values due to the fabrication tolerances. Note that all the analytical results presented in this paper are obtained while considering the Young's modulus of $169 \mathrm{GPa}$ and Poisson's ratio of 0.27 for silicon based nano beams.

\begin{tabular}{|c|c|c|c|c|}
\hline $\begin{array}{c}\text { Length } \\
(\boldsymbol{\mu m})\end{array}$ & $\begin{array}{c}\text { depth } \\
(\mathbf{n m})\end{array}$ & $\begin{array}{c}\text { Thickness } \\
(\mathbf{n m})\end{array}$ & $\begin{array}{c}\text { Gap } \\
(\mathbf{n m})\end{array}$ & $\begin{array}{c}\text { Initial rise } \\
(\mathbf{n m})\end{array}$ \\
\hline 15 & 850 & 70 & 850 & 122 \\
\hline 15 & 850 & 120 & 850 & 116 \\
\hline 15 & 850 & 70 & 1000 & 123 \\
\hline 15 & 850 & 120 & 1000 & 97.3 \\
\hline
\end{tabular}

Table I. Effective dimensions used for obtaining the analytical results.

The experimental results show an increase in the resonance frequency of the arches with an increase in the DC bias voltage. This is due to the increase in the stiffness of the arch with the increasing axial force due to the applied voltage. The arches shows tunability of $77.12 \%$ and $85.51 \%$ under DC biased conditions with $850 \mathrm{~nm}$ and $1 \mu \mathrm{m}$ transduction gap between the beam and the driving/sensing electrodes, respectively. A closer look towards the tunability of the two arches under study with gaps of $850 \mathrm{~nm}$ and $1 \mu \mathrm{m}$ reveals an increase of $56.29 \%$ and $66.07 \%$, respectively for DC bias changes from $40 \mathrm{~V}$ to $110 \mathrm{~V}$. This is attributed to the dominating effect of mid-plane stretching over the softening effect of the electrostatic force for large gaps, as previously found in [10] and [16].

Similarly, the resonance frequencies of other two nano arches of $15 \mu \mathrm{m}$ long, $850 \mathrm{~nm}$ deep, and $180 \mathrm{~nm}$ thick with transduction gaps of $850 \mathrm{~nm}$ and $1 \mu \mathrm{m}$ are found to increase with increasing DC polarization voltages, Fig. 7a and Fig. 7b. The increase in resonance frequency of these shallow arches is also due to the increase in the stiffness of the arch with increasing axial force due to applied DC voltage showing a tunability of $73.05 \%$ and $108.14 \%$ with $850 \mathrm{~nm}$ and $1 \mu \mathrm{m}$ transduction gap between the beam and the driving/sensing electrodes, respectively. Tunability of $34.71 \%$ and $45.14 \%$ are achieved for the arches with $850 \mathrm{~nm}$ and $1 \mu \mathrm{m}$ transduction gap, respectively, with $\mathrm{DC}$ bias changes from $40 \mathrm{~V}$ to $110 \mathrm{~V}$ again reflects the dominating effect of mid-plane stretching over electrostatic force for large gaps.

Therefore, thicker arches can achieve higher tunability compared to the thin arches due to the relatively higher pull-in voltages. However, the thinner beams show higher tunability per unit increase in the DC polarization voltage due to the combined effect of increasing arch stiffness and dominating effect of mid-plane stretching over electrostatic force for large gaps. The practical realization of these arches open up the possibility to use them for tunable band pass filtering for TV tuner applications with the flexibility of adjustable bandwidth by coupling the nanomechanical arches.

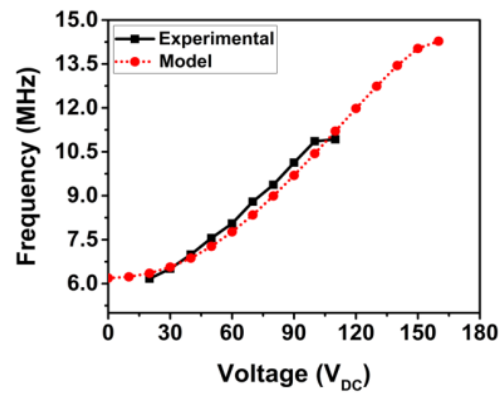

(a)

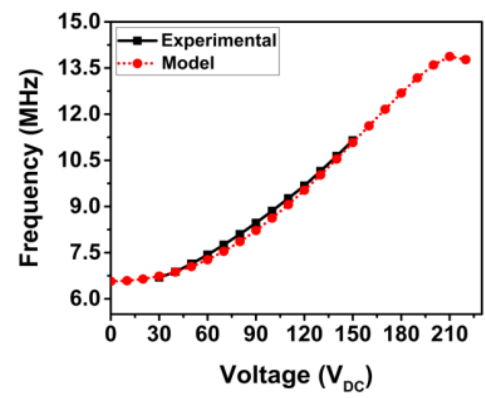

(b)

Fig. 6: The DC bias volatge versus resonance frequency for shallow arches of $15 \mu \mathrm{m}$ length, $850 \mathrm{~nm}$ depth and $130 \mathrm{~nm}$ thickness with a transduction gap of (a) $850 \mathrm{~nm}$ (b) $1 \mu \mathrm{m}$.

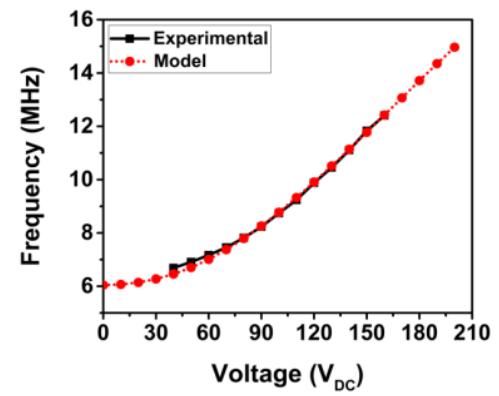

(a)

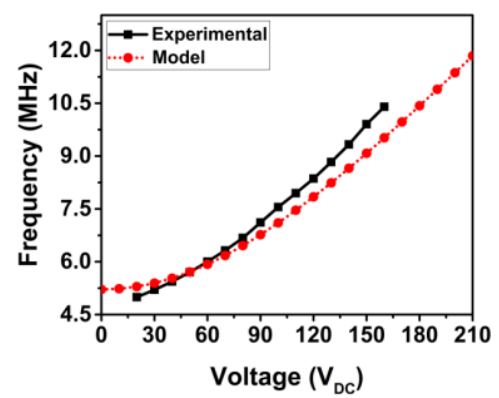

(b)

Fig. 7: The DC bias volatge versus resonance frequency for shallow arches of $15 \mu \mathrm{m}$ length, $850 \mathrm{~nm}$ depth and $180 \mathrm{~nm}$ thickness with a transduction gap of (a) $850 \mathrm{~nm}$ (b) $1 \mu \mathrm{m}$. 


\section{CONCLUSIONS}

In conclusions, an analytical and experimental study is presented on the tunability of in-plane doubly-clamped nanomechanical arches under varied DC bias conditions at room temperature. The experimental results show good agreement with that of the analytical ones for the arches with carefully designed gap to thickness ratios. Therefore, the arches show high tunability by electrostatic actuation. The high tunability of shallow arch open up the possibilities for tunable band pass filtering application in high frequency range (3-30 $\mathrm{MHz})$.

\section{ACKNOWLEDGMENTS}

This work has been supported through King Abdullah University of Science and Technology (KAUST) research funds.

\section{REFERENCES}

[1] Park, K., Kim, N., Morisette, D. T., Aluru, N., Bashir, R., "Resonant MEMS mass sensors for measurement of microdroplet evaporation", Journal of Microelectromechanical Systems, 21(3), 702-711, 2012.

[2] Burg, T. P., Mirza, A. R., Milovic, N., Tsau, C. H., Popescu, G., Foster, J. S., Manalis, S. R., "Vacuumpackaged suspended microchannel resonant mass sensor for biomolecular detection", Journal of Microelectromechanical Systems, 15(6), 1466-1476, 2006.

[3] Al Hafiz, M. A., Kosuru, L., and Younis, M.I., "Electrothermal Frequency Modulated Resonator for Mechanical Memory", Journal of Microelectromechanical Systems, 25(5), 877-883, 2016.

[4] Al Hafiz, M. A., Kosuru, L., and Younis, M. I., "Microelectromechanical reprogrammable logic device", Nature communications, 7, 2016.

[5] Nilchi, J. N., Liu, R., and Nguyen, C. T. -C, "7th order sharp-roll-off bridged micromechanical filter", International Solid-State Sensors, Actuators and Microsystems Conference, 2015.

[6] Nguyen, C. T. -C, "Frequency-selective MEMS for miniaturized low-power communication devices", IEEE Transactions on Microwave Theory and Techniques, 47(8), 1486-1503, 1999.

[7] Dellea, S., Giacci, F., Longoni, A. F., and Langfelder, G., "In-plane and out-of-plane mems gyroscopes based on piezoresistive nems detection", Journal of Microelectromechanical Systems, 24(6), 1817-1826, 2015.

[8] Altena, G., Hohlfeld, D., Elfrink, R., Goedbloed, M. H., and Schaijk, R. Van., "Design, modeling, fabrication and characterization of an electret-based MEMS electrostatic energy harvester", International Solid-State Sensors,
Actuators and Microsystems Conference, 2011.

[9] Lin, L., Howe, R. T. and Pisano, A. P. "Microelectromechanical filters for signal processing", Journal of Microelectromechanical Systems, 7(3), 286-294, 1998.

[10] Abdel-Rahman, E. M., Younis, M. I., and Nayfeh, A. H., "Characterization of the mechanical behavior of an electrically actuated microbeam", Journal of Micromechanics and Microengineering, 12(6), 759, 2002.

[11] Maekoba, H., Helin, P., Reyne, G., Bourouina, T., and Fujita, H., "Self-aligned vertical mirror and V-grooves applied to an optical-switch: modeling and optimization of bi-stable operation by electromagnetic actuation", Sensors and Actuators A: Physical, 87(3), 172-178, 2001.

[12]Hajjaj, A. Z., Alcheikh, N., Ramini, A., Al Hafiz, M. A., and Younis, M. I., "Highly tunable electrothermally and electrostatically actuated resonators", Journal of Microelectromechanical Systems, 25(3), 440-449, 2016.

[13] Nathanson, H. C., Newell, W. E., Wickstrom, R. A., and Davis, J. R., "The resonant gate transistor", IEEE Transactions on Electron Devices 14(3), 117-133, 1967.

[14] Younis, M. I., Miles, R. and Jordy, D., "Investigation of the response of microstructures under the combined effect of mechanical shock and electrostatic forces", Journal of Micromechanics and Microengineering, 16(11), 2463, 2006.

[15] Nayfeh, A. H., Younis, M. I., and Abdel-Rahman, E. M., "Reduced-order models for MEMS applications", Nonlinear dynamics, 41(1), 211-236, 2005.

[16]Hajjaj, A. Z., Ramini, A. and Younis, M. I., "Experimental and analytical study of highly tunable electrostatically actuated resonant beams", Journal of Micromechanics and Microengineering, 25(12), 125015, 2015.

[17] Sazonova, V., Yaish, Y., Üstünel, H., Roundy, D., Arias, T. A., and McEuen, P. L., "A tunable carbon nanotube electromechanical oscillator", Nature, 431, 284-287, 2004.

[18] Younis. M. I., "MEMS Linear and Nonlinear Statics and Dynamics", Vol. 20. New York, NY, USA: Springer, 2011. 